\title{
EVALUACIÓN Y PROPUESTAS DE MEJORA DEL USO DE LA ENERGÍA EN REDES DE RIEGO. APLICACIÓN A LA COMUNIDAD DE REGANTES MURADA NORTE (ORIHUELA, ALICANTE)
}

\author{
Ruiz-Rodríguez, M. (1) (P), Jiménez-Bello, M.A. (2), Pulido-Velázquez, M. (3)
}

\begin{abstract}
1 Candidato a Máster Universitario en Ingeniería de Caminos, ETSI Caminos, Canales y Puertos, Universitat Politècnica de València, Camino de Vera s/n, 46022 Valencia, Spain, marruir2@gmail.com

${ }^{2}$ Profesor, Instituto universitario de investigación de Ingeniería del Agua y Medio Ambiente (IIAMA), Universitat Politècnica de València, Camino de Vera s/n, 46022 Valencia, Spain, mijibar@dihma.upv.es

${ }^{3}$ Profesor, Instituto universitario de investigación de Ingeniería del Agua y Medio Ambiente (IIAMA), Universitat Politècnica de València, Camino de Vera s/n, 46022 Valencia, Spain, mapuve@upv.es
\end{abstract}

\section{Resumen}

La modernización de las infraestructuras agrícolas de regadío ha supuesto generalmente un incremento en el consumo energético de estas instalaciones. Tomando como referencia el Protocolo de Auditoría Energética en Comunidad de Regantes (CCRR) del Instituto para la Diversificación y Ahorro de Energía (IDAE), se evalúan e identifican actuaciones de mejora del uso de la energía en la red de riego de la CCRR Murada Norte (Orihuela, Alicante). En primer lugar se caracteriza la red de riego, su gestión y su consumo, elaborando un modelo matemático de la red con herramientas SIG y EPANET. Posteriormente se realiza una evaluación del uso de la energía con los indicadores de gestión energética del protocolo del IDAE, a través del cual se consigue calificar energéticamente la CCRR. Finalmente como actuaciones de mejora se propone la construcción de una nueva balsa de regulación que permite reducir la altura media de impulsión, la sustitución del equipo de bombeo actual para mejorar la eficiencia del bombeo y reducir la potencia contratada en horas punta, y la renegociación de la tarifa eléctrica.

\section{1- Introducción}

En España la agricultura de regadío produce casi el $60 \%$ de la producción agrícola nacional, que representa alrededor del $8 \%$ del PIB nacional, utilizando únicamente el $14 \%$ de la superficie agraria útil (Del Campo, 2014). Esto justifica la gran importancia económica del regadío dentro el sector de la agricultura en nuestro país. El regadío es el uso consuntivo que más agua demanda, tanto en España como a nivel mundial $(70 \%)$ (WWAP, 2014). Por esta razón, durante los últimos años se viene produciendo una modernización de las técnicas de riego en este sector de acuerdo con el Plan Nacional de Regadíos, con el objetivo de realizar un uso más eficiente del recurso hídrico y cuya inversión ha supuesto unos 6.000 millones de euros (Del Campo, 2014).

Los resultados se reflejan en datos del INE, donde se observa que la demanda de años atrás de $24.250 \mathrm{Hm} 3$ anuales, representando un $80 \%$ de los usos consuntivos, ha pasado a partir del proceso de modernización al entorno de los $16.000 \mathrm{Hm} 3$, representando 
un $63 \%$ de los usos consuntivos. Esta evolución ha sido posible gracias a la mayor utilización de técnicas de riego más eficientes con el uso del agua, destacando el riego localizado, comúnmente conocido como riego por goteo. Actualmente, España es el segundo país del mundo en porcentaje de uso de riego localizado, y el primero en número de hectáreas regadas por este método (Del Campo, 2014). Esta tendencia a un mayor uso del riego localizado ha supuesto un aumento del consumo energético en las instalaciones de regadío, ya que estas técnicas requieren de una mayor aportación de energía a la red en forma de presión. "La mayor eficiencia hídrica va ligada a una mayor ineficiencia energética" (Corominas, 2010). En 2010 la agricultura de regadío era responsable del 2,5\% del consumo energético total del país (Hardy y Garrido, 2010). En consecuencia, el coste en término de potencia de la factura eléctrica en las Comunidades de Regantes (CCRR), coste fijo independiente del consumo, ha aumentado entre el $1000 \%$ y el $1200 \%$ entre los años 2008 y 2013, siendo el incremento total de la factura eléctrica en este período de aproximadamente el 100\% (Del Campo, 2014). En relación con estos aspectos, y buscando reducir las emisiones de Gases Efecto Invernadero (GEI) de acuerdo con el Protocolo de Kioto, el Instituto para la Diversificación y Ahorro de la Energía (IDAE) con la colaboración del MAGRAMA elaboraron en 2008 una serie de documentos técnicos en materia de eficiencia energética en Comunidades de Regantes: "Ahorro y Eficiencia Energética en las Comunidades de Regantes" y "Protocolo de Auditoría Energética en Comunidades de Regantes".

En este trabajo, tomando como referencia ambos documentos del IDAE, se evalúan e identifican actuaciones de mejora del uso de la energía en la red de riego de la CCRR Murada Norte, situada en el término municipal de Orihuela (Alicante).

\section{2- Materiales y métodos}

El procedimiento llevado a cabo para identificar y analizar actuaciones que permitan un mejor uso energético de la red de riego se compone de tres fases: Caracterización de la red, Evaluación del uso de la energía y Análisis de las propuestas de mejora.

\subsection{Caracterización de la red}

En la fase de caracterización de la red, se recopila información relativa a los consumos de agua y energía y su distribución temporal durante el período analizado, los elementos que componen la red, y el modelo de gestión y explotación de la instalación. Con esta información, y haciendo uso de Sistemas de Información Geográfica (SIG) y el programa de simulación de redes presurizadas EPANET, se elabora un modelo matemático de la red que permite analizar su comportamiento hidráulico y simular escenarios distintos al actual (Jiménez, 2008).

\subsection{Evaluación del uso de la energía}

En la fase de evaluación del uso de la energía, se utilizan indicadores de gestión energética que permiten cuantificar y calificar el uso de la energía. En este estudio se utilizan los indicadores propuestos en el Protocolo de Auditoría Energética del IDEA (IDAE, 2008). Los indicadores más destacados son:

- IDE - Índice de Dependencia Energética (\%): Representa el porcentaje de agua que es bombeado frente al total de agua que entra en la CR. Se calcula como: 
$\operatorname{IDE}=\frac{\mathrm{V}_{\mathrm{B}}}{\mathrm{V}_{0}}$

- $\quad V_{B}=$ Volumen total bombeado.

- $\quad V_{0}=$ Volumen total que entra al sistema.

- ICE - Índice de Carga Energética $(\mathrm{m})$ : Representa la altura manométrica media suministrada por los bombeos. Se calcula como:

ICE $=\frac{\sum \mathrm{V}_{\mathrm{B}, \mathrm{i}} \cdot \mathrm{H}_{\mathrm{i}}}{\mathrm{V}_{0}}$

- $\quad V_{B, i}=$ Volumen impulsado por el grupo de bombeo i.

- $H_{\mathrm{i}}=$ Presión manométrica aportada por el grupo de bombeo i $(\mathrm{m})$.

- $\quad V_{0}=$ Volumen que entra al sistema.

- EEB - Eficiencia Energética de Bombeos (\%): Representa el porcentaje de potencia absorbida que se transmite al fluido en forma de potencia hidráulica. Se puede calcular según la siguiente fórmula, tanto para un grupo de bombeo $\left(E E B_{i}\right)$ como para el total de la instalación (EEB):

$$
\begin{aligned}
& \mathrm{EEB}_{i}=\frac{\gamma \cdot \mathrm{V}_{\mathrm{B}, \mathrm{i}} \cdot \mathrm{H}_{\mathrm{i}}}{3600 \cdot \mathrm{E}_{\mathrm{i}}} \\
& \mathrm{EEB}=\frac{\sum \mathrm{EEB}_{\mathrm{i}} \cdot \mathrm{E}_{\mathrm{i}}}{\mathrm{E}}=\frac{\gamma \cdot \sum \mathrm{V}_{\mathrm{B}, \mathrm{i}} \cdot \mathrm{H}_{\mathrm{i}}}{3600 \cdot \mathrm{E}}=\frac{\gamma \cdot \mathrm{V}_{0} \cdot \mathrm{ICE}}{3600 \cdot \mathrm{E}}
\end{aligned}
$$

- $\quad \gamma=$ Peso específico del fluido $\left(9,8\right.$ en el caso del agua) $\left(\mathrm{kN} / \mathrm{m}^{3}\right)$.

- $\quad V_{B, i}=$ Volumen impulsado por el grupo de bombeo $i\left(\mathrm{~m}^{3}\right)$.

- $H_{\mathrm{i}}=$ Presión manométrica aportada por el grupo de bombeo i $(\mathrm{m})$.

- $\quad E_{i}=$ Energía consumida por el grupo de bombeo $i(k W h)$.

- $\quad E=$ Energía total consumida por los bombeos de la instalación (kWh).

- $V_{0}=$ Volumen total que entra al sistema $\left(\mathrm{m}^{3}\right)$.

- $\quad$ ICE = Índice de carga energética (m).

- ESE - Eficiencia de Suministro Energético (\%): Representa el cociente entre la energía necesaria a aportar al sistema y la energía real aportada. Se puede calcular como:

$$
\begin{aligned}
& \text { ESE }=\frac{\Delta E}{I C E}=\frac{\sum\left(Z_{j}+P_{j}\right) \cdot S_{j}}{I C E \cdot S}-H_{0} \\
& H_{0}=\frac{\sum\left(Z_{k}+P_{k}\right) \cdot V_{k}}{V_{0}} \\
& -\quad Z_{j}=\text { Cota del hidrante } j(m) . \\
& -\quad P_{j}=\text { Presión manométrica requerida en el hidrante } j(m) . \\
& -\quad S_{j}=\text { Superficie regada por el hidrante } j . \\
& -\quad Z_{k}=\text { Superficie regada total. }
\end{aligned}
$$


- $\quad P_{k}=$ Presión manométrica en el punto de captación $\mathrm{k}(\mathrm{m})$.

- $\quad V_{k}=$ Volumen del punto de captación $k$ que entra al sistema.

- $\quad V_{0}=$ Volumen total que entra al sistema.

- $\quad$ ICE = Índice de carga energética (m).

- EEG - Eficiencia de Energética General de la CCRR (\%): Representa la eficiencia energética general de la red de distribución de toda la CCRR. Se calcula como:

$$
E E G=E E B \cdot E S E
$$

- $\quad E E B=$ Eficiencia energética de bombeos $(\%)$.

- $\quad E S E=$ Eficiencia de suministro energético (\%).

- PME - Precio Medio de la Energía (€/kWh): Representa el cociente entre el gasto energético final (incluyendo todos los términos) y el consumo energético. Es un indicador que permite comparar distintas tarifas eléctricas y/o calendarios de funcionamiento.

\subsection{Análisis de las propuestas de mejora}

Las propuestas de mejora tienen como objetivo reducir la dependencia energética (IDE), maximizar la eficiencia energética de la instalación (EEG) o minimizar su coste (PME). La mejora de la EEG supone incrementar la eficiencia de los bombeos (EEB), o mejorar la eficiencia de suministro (ESE), lo que implica reducir la carga energética (ICE).

Identificada una actuación que permita mejorar alguno de los indicadores mencionados se analiza su viabilidad económica, mediante la obtención de los beneficios económicos y la estimación de sus costes de ejecución. Para ello se obtienen indicadores de rentabilidad económica, como el VAN, que permiten justificar la viabilidad económica de la actuación y comparar entre distintas propuestas.

\subsection{Caso de estudio}

El procedimiento descrito se ha aplicado en la red de riego de la CCRR Murada Norte. Esta instalación cuenta con una dotación hídrica anual de $2 \mathrm{hm}^{3}$ procedentes del canal del trasvase Tajo-Segura, regando una superficie de 1.000 ha. La red consta de un grupo de bombeo de captación, que impulsa el agua desde el canal del trasvase hasta dos balsas de regulación, situadas $115 \mathrm{~m}$ por encima del punto de captación (cota $125 \mathrm{msnm}$ ). De las balsas de regulación se abastece a los comuneros mediante dos redes de distribución independientes, una de ellas utilizando un segundo bombeo y la otra directamente por gravedad. En la siguiente figura (Figura 1) se representan en un mapa las dos redes de distribución. 

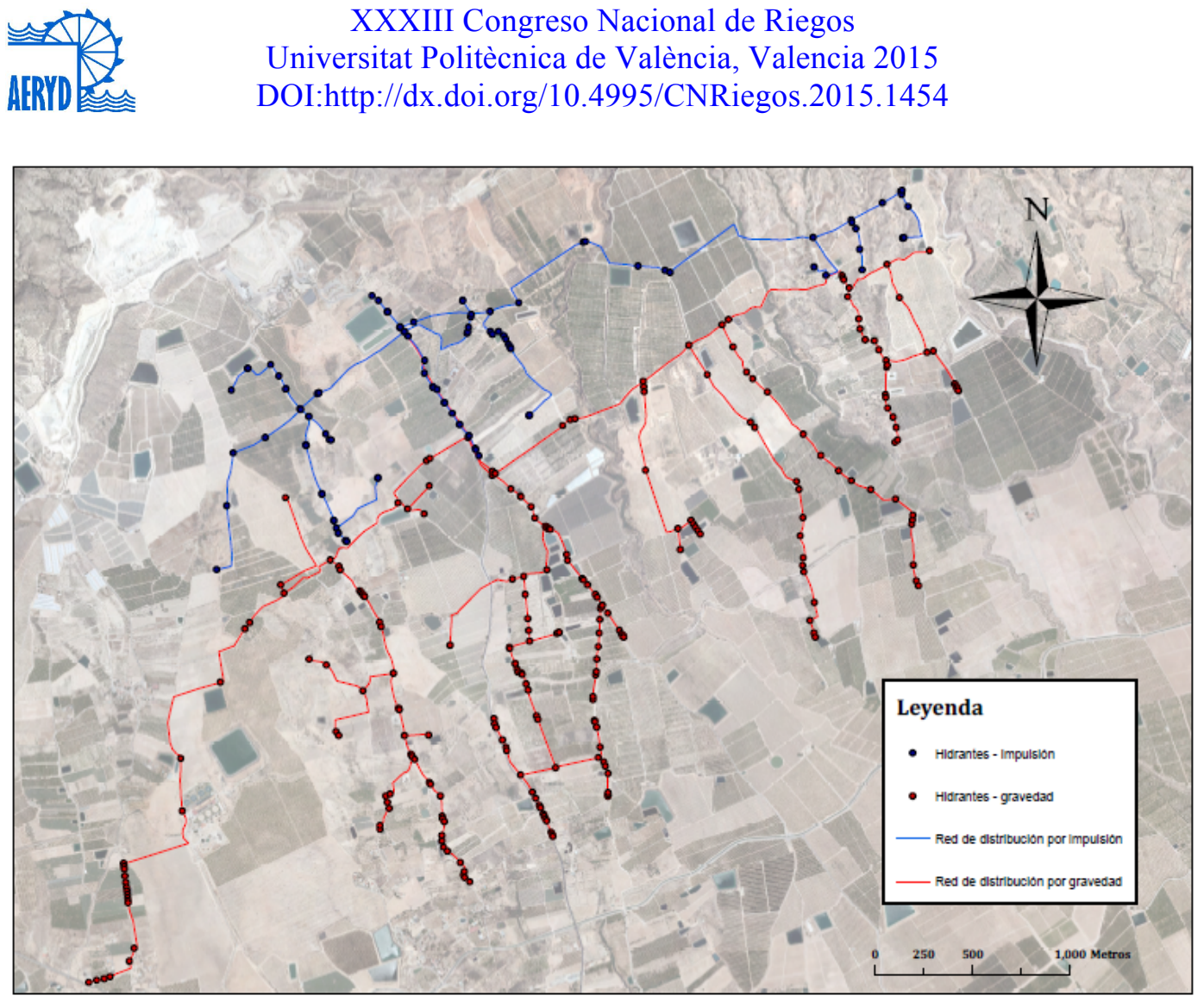

Figura 1. Mapa de la CCRR en el que se representan la red de distribución (rojo) por gravedad y la red de distribución con impulsión (azul).

La estación de bombeo de captación consta de un equipo principal de $295 \mathrm{~kW}$ y un segundo equipo de reserva de $215 \mathrm{~kW}$. La altura manómetrica media de este grupo de bombeo es de $125 \mathrm{~m}$. La tubería de impulsión hasta las balsas es DN $500 \mathrm{~mm}$ de Fibrocemento. La capacidad de regulación total de las balsas es de $228.000 \mathrm{~m}^{3}$, con una cota media de la lámina de agua de $236 \mathrm{msnm}$. La mayor parte de la red de distribución es de PVC. Aproximadamente $2 / 3$ de la superficie se riegan con la red de distribución por gravedad y el restante $1 / 3$ con la red que requiere una segunda impulsión. La estación de bombeo de abastecimiento en baja consta de tres grupos de bombeo que funcionan con velocidad variable, dos de ellos de $90 \mathrm{~kW}$ y el tercero de $15 \mathrm{~kW}$. Estos equipos de bombeo suministran una presión de $30 \mathrm{~m}$. Finalmente la presión que se requiere en hidrantes es de $25 \mathrm{~m}$. Con esta información se han realizado los modelos de ambas redes de distribución (Figura 2).
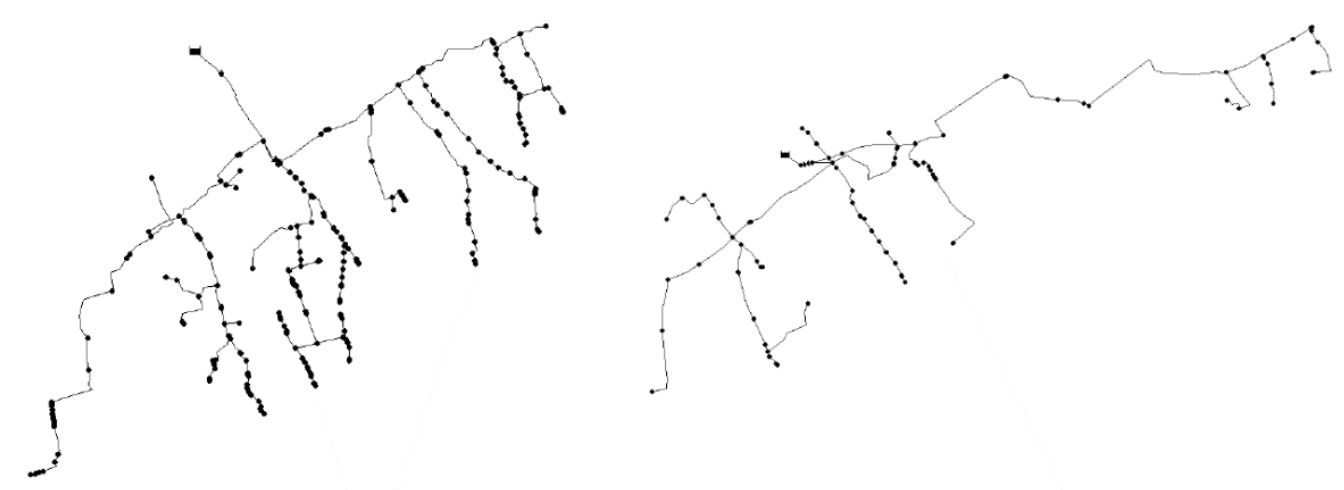
Figura 2. Modelos en EPANET de las redes de distribución por gravedad (izquierda) y por impulsión (derecha).

El perído de análisis adoptado en este trabajo es un año, comprendiendo el consumo energético durante el año 2013. En este período se consumen un total de 1.300.851 kWh en la estación de bombeo en alta (bombeo de captación), y 36.972 kWh (3\% del consumo total) en la estación de bombeo en baja. La tarifa eléctrica contratada es la 3.1. A, con discriminación horaria en tres períodos: punta, llano y valle. En los siguientes gráficos (Figura 3) se representan la distribución mensual del consumo energético de ambas estaciones de bombeo, distinguiendo qué cantidad corresponde a cada período tarifario.
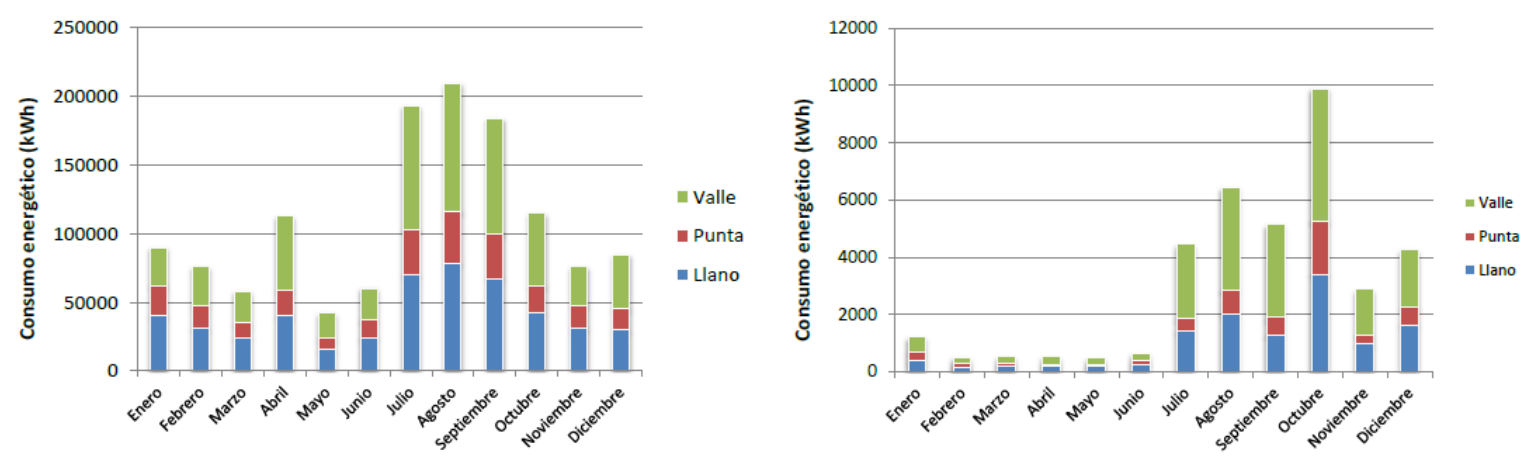

Figura 3. Evolución del consumo energético de la estación de bombeo en alta (izquierda) y en baja (derecha) durante el año 2013.

\section{3- Resultados y discusión}

\subsection{Calificación energética}

En la siguiente tabla (Tabla 1) se resumen los valores de los indicadores de gestión energética aplicados a la red de riego analizada.

Tabla 1. Valores de los principales indicadores de gestión energética.

\begin{tabular}{lc}
\hline INDICADOR & VALOR \\
\hline IDE: Índice de dependencia energética (\%) & 100,00 \\
ICE: Índice de carga energética (m) & 136,65 \\
EEB: Eficiencia energética de bombeos (\%) & 56,84 \\
ESE: Eficiencia de suministro energético (\%) & 64,22 \\
EEG: Eficiencia energética general de la CCRR (\%) & 36,50 \\
PME: Precio medio de la energía (cts€/kWh) & 14,98 \\
\hline
\end{tabular}

De acuerdo con el protocolo de auditoría energética en CCRR del IDAE, se obtiene una calificación energética de C (Eficiencia Normal). La calificación de la eficiencia del bombeo también sería de $\mathrm{C}$ (Normal). 


\subsection{Construcción de una nueva balsa de regulación}

Gracias a la simulación con EPANET se observa que se producen presiones superiores a los $60 \mathrm{mca}$ en casi el $50 \%$ de la superficie regada, valor que no es recomendable sobrepasar en este tipo de instalaciones. Parte de esta excesiva presión es disipada por Válvulas Reductoras de Presión colocadas en la red de distribución por gravedad, lo cual constituye una ineficiencia energética reflejada en un menor valor de ESE.

Con el objetivo de aumentar el ESE (reducir el ICE) y reducir las presiones producidas en la red se plantea la construcción de una nueva balsa de regulación a menor cota de las ya existentes. Además de la construcción de la balsa, esta actuación requerirá colocar un nuevo grupo de bombeo y una nueva tubería de conexión con la captación y con la estación de filtrado de cabecera. De entre distintas posibilidades de ubicación de la balsa se concluye que la alternativa para la que se obtienen mayores beneficios es la construcción de una balsa de $38.500 \mathrm{~m}^{3}$ con la lámina de agua a la cota $204 \mathrm{msnm}$.

Tabla 2. Indicadores económicos de la construcción de la nueva balsa.

\begin{tabular}{lc}
\hline INDICADOR & VALOR \\
\hline Costes de inversión $(€)$ & 1.068 .884 \\
Beneficios anuales (€/año) & $25.585,35$ \\
Coste de inversión/Beneficios anuales (años) & 41,78 \\
VAN (25 años con tasa de interés del 4\%)(€) & $-669.187,62$ \\
\hline
\end{tabular}

Al obtener un VAN negativo se concluye que la propuesta de mejora no resulta rentable económicamente. Sin embargo esta actuación quedaría justificada en el caso en que sea necesaria la construcción de una nueva balsa por necesidades de regulación. Además permite reducir la presión significativamente en los puntos más bajos de la red, como se observa en la Figura 4, lo que supondría unos beneficios en mantenimiento no contabilizados.
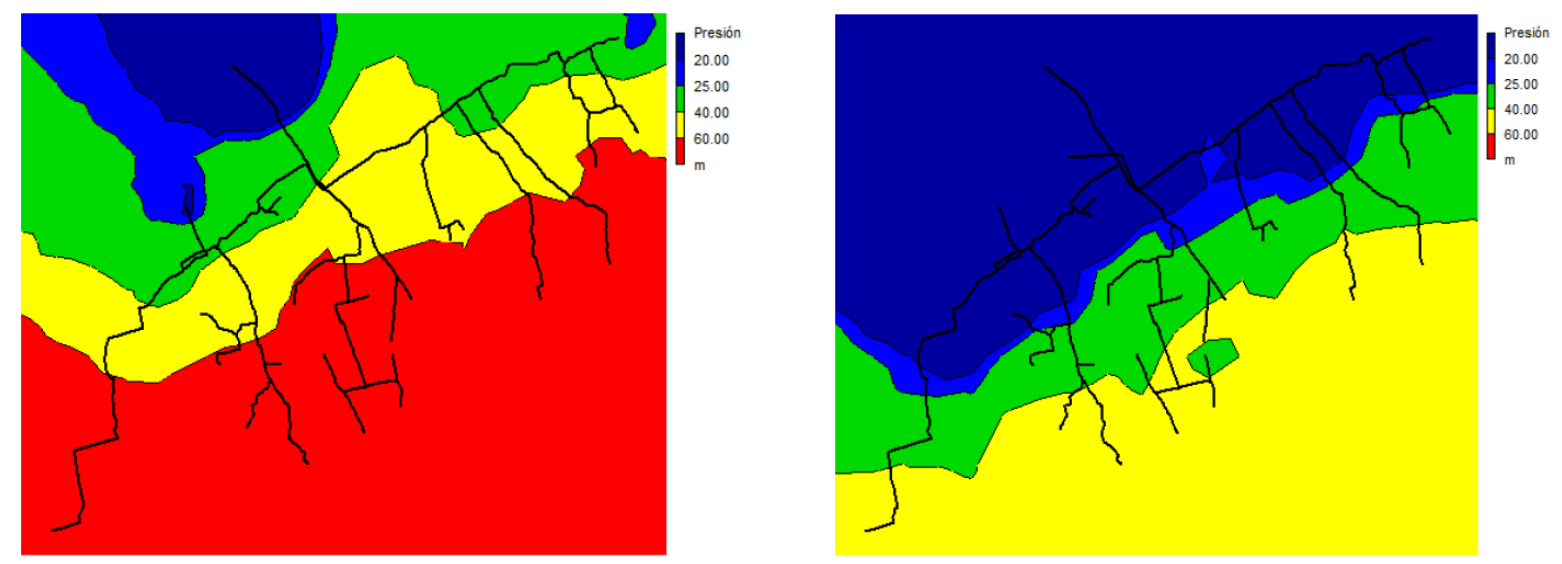

Figura 4. Simulación de las curvas de presión con el modelo de EPANET antes de la actuación (izquierda) y después (derecha). 


\subsection{Nuevos equipos de bombeo para el abastecimiento en alta}

Con el objetivo de reducir la potencia contratada en período punta, donde la tarifa de acceso es un $700 \%$ mayor que en período valle (2014), se plantea añadir un nuevo grupo de menos potencia en la estación de bombeo en alta, de manera que sólo este equipo trabaje en puntas, y conjuntamente con el equipo principal en llano y valle. Con esta condición se obtiene un caudal mínimo necesario de 25,39 I/s. Se selecciona una bomba multicelular APM 100/6 de $60 \mathrm{~kW}$ (Bombas IDEAL 2014), capaz de suministrar $32 \mathrm{l} / \mathrm{s}$ a $125 \mathrm{~m}$. En la siguiente tabla (Tabla 3) se observan los resultados del análisis económico.

Tabla 3. Indicadores económicos de la instalación del equipo de bombeo de 60 kW.

\begin{tabular}{lc}
\hline INDICADOR & VALOR \\
\hline Costes de inversión $(€)$ & 22.800 \\
Beneficios anuales (€/año) & $16.524,28$ \\
Coste de inversión/Beneficios anuales (años) & 1,38 \\
VAN (20 años con tasa de interés del 4\%) (€) & $201.770,36$ \\
\hline
\end{tabular}

La estación de bombeo en alta presenta un EEB de 56,71\% (Eficiencia Normal). Puesto que se trata de un bombeo que trabaja en un punto de funcionamiento (PF) fijo, lo ideal sería que el PF coincidiera con la máxima eficiencia del bombeo. Con el objetivo de mejorar el EEB se plantea la sustitución del equipo principal de la estación de bombeo en alta por un equipo que ofrezca una mayor eficiencia energética para el PF objetivo (125 m). En este caso se selecciona una bomba multicelular APM 200/2 con una eficiencia total del $72 \%$ y una potencia de $240 \mathrm{~kW}$ (Bombas IDEAL 2014), capaz de suministrar $140 \mathrm{l} / \mathrm{s}$ a $125 \mathrm{~m}$. A continuación (Tabla 4) se observan los indicadores de viabilidad económica.

Tabla 4. Indicadores económicos de la instalación del equipo de bombeo de $240 \mathrm{~kW}$.

\begin{tabular}{lc}
\hline INDICADOR & VALOR \\
\hline Costes de inversión $(€)$ & 94.400 \\
Beneficios anuales (€/año) & 29.351 \\
Coste de inversión/Beneficios anuales (años) & 3,22 \\
VAN (20 años con tasa de interés del 4\%) (€) & $304.489,53$ \\
\hline
\end{tabular}

\subsection{Contratación de la tarifa 6.1.}

La principal ventaja de esta tarifa radica en que durante el mes de agosto, mes de mayor consumo energético en el caso de estudio, se aplica el período tarifario valle. Realizando una estimación de costes se obtienen unos beneficios aproximados de 42.818 €/año (impuestos incluidos) con esta tarifa, según datos de facturación de 2013 y tarifa de acceso de 2014.

\subsection{Análisis comparativo del conjunto de mejoras propuestas}

Por tanto, para mejorar el uso de la energía en el caso analizado se proponen las siguientes cuatro actuaciones: 
- Con el objetivo de aumentar el ESE se plantea la construcción de una nueva balsa de regulación.

- Para reducir la potencia contratada en horario punta se plantea la colocación de un nuevo grupo de bombeo en la estación de captación.

- Con la finalidad de incrementar el EEB se plantea la sustitución del grupo principal de la estación de bombeo en alta.

- Con el objetivo de reducir el PME se propone un cambio a la tarifa 6.1.

En la Tabla 5 se comparan los indicadores iniciales con los obtenidos al aplicar todas las mejoras y al aplicar todas salvo la construcción de la nueva balsa.

Tabla 5. Comparación de los indicadores de gestión energética antes de las mejoras, aplicando todas las mejoras, y aplicando todas las mejoras menos la nueva balsa.

\begin{tabular}{|c|c|c|c|}
\hline INDICADOR & $\begin{array}{l}\text { SITUACIÓN } \\
\text { INICIAL }\end{array}$ & $\begin{array}{l}\text { TODAS LAS } \\
\text { MEJORAS }\end{array}$ & $\begin{array}{l}\text { MEJORAS SIN } \\
\text { BALSA }\end{array}$ \\
\hline $\begin{array}{l}\text { IDE: Índice de dependencia } \\
\text { energética }(\%)\end{array}$ & 100,00 & 100,00 & 100,00 \\
\hline ICE: Índice de carga energética (m) & 136,65 & 124,59 & 136,65 \\
\hline $\begin{array}{l}\text { EEB: Eficiencia energética de } \\
\text { bombeos }(\%)\end{array}$ & 56,84 & 67,9 & 69,1 \\
\hline $\begin{array}{l}\text { ESE: Eficiencia de suministro } \\
\text { energético }(\%)\end{array}$ & 64,22 & 70,43 & 64,22 \\
\hline $\begin{array}{l}\text { EEG: Eficiencia energética general de } \\
\text { la CCRR }(\%)\end{array}$ & 36,50 & 47,82 & 44,38 \\
\hline $\begin{array}{l}\text { PME: Precio medio de la energía } \\
\text { (cts } € / \mathrm{kWh} \text { ) }\end{array}$ & 14,98 & 9,94 & 10,66 \\
\hline $\begin{array}{l}\text { E: Consumo energético total de la } \\
\text { CCRR (kWh/año) }\end{array}$ & 1.337 .823 & 866.699 & 1.047 .994 \\
\hline Inversión inicial $(€)$ & 0 & 1.186 .084 & 117.200 \\
\hline $\begin{array}{l}\text { VAN medio anual ( } 20 \text { años con tasa } \\
\text { de interés del } 4 \% \text { ( } € / \text { año) }\end{array}$ & 0 & 18.350 & $54.408,54$ \\
\hline Calificación Energética del Bombeo & $\mathrm{C}$ & $A$ & $A$ \\
\hline Calificación Energética de la CCRR & $\mathrm{C}$ & B & $\mathrm{B}$ \\
\hline Grupo de Consumo Energético & $\begin{array}{c}\text { Gran } \\
\text { Consumidora }\end{array}$ & Consumidora & $\begin{array}{c}\text { Gran } \\
\text { Consumidora }\end{array}$ \\
\hline
\end{tabular}

Como se puede comprobar, con todas las mejoras propuestas se estima un ahorro energético del $35 \%$ sobre el consumo inicial ( $471 \mathrm{MWh} / \mathrm{año})$, mejorando la calificación 


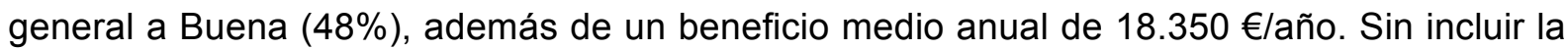
construcción de la nueva balsa en el paquete de mejoras, se obtienen beneficios anuales medios mucho mayores con muy poca inversión, $54.409 € /$ año con una inversión inicial de tan solo $117.200 €$, aunque el ahorro energético es menor con un $22 \%$ sobre el consumo inicial (290 MWh/año).

\section{4- Conclusiones}

La caracterización de la red de riego y la aplicación de los indicadores de gestión energética propuestos en el Protocolo de Auditoría Energética en CCRR ha permitido calificar el consumo de la CCRR Murada Norte. Con el objetivo de mejorar estos indicadores se plantean actuaciones que han permitido reducir el consumo y coste energético de la CCRR.

El procedimiento llevado a cabo en este estudio, aplicable en otras CCRR, resulta ser una herramienta útil para alcanzar una gestión eficiente de estas instalaciones, permitiendo el planteamiento de alternativas de actuación con repercusiones ambientales y económicas favorables.

\section{5- Agradecimientos}

Los autores de este trabajo muestran su agradecimiento a la CCRR Murada Norte por su participación en este estudio y al proyecto IMPADAPT (CGL2013-48424-C2-1-R) del Plan Estatal de I+D+i (Ministerio de Economía y Competitividad) por su financiación.

\section{6.- Bibliografía}

Corominas, J. (2010). Agua y energía en el riego, en la época de la sostenibilidad. Ingeniería del Agua, 17, 219-233.

Del Campo, A. (2014). El factor de la energía en los grandes usuarios del agua. Día Mundial del Agua 2014. Sede del Canal Isabel II, Madrid.

Hardy L. y Garrido A. (2010). Análisis y Evaluación de las Relaciones entre el Agua y la Energía en España. Fundación Botín, Madrid.

IDAE (2008). Ahorro y Eficiencia Energética en las Comunidades de Regantes. Instituto para la Diversificación y Ahorro de Energía, Madrid: Ministerio de Industria, Comercio y Turismo.

IDAE (2008). Protocolo de Auditoría Energética en Comunidades de Regantes. Instituto para la Diversificación y Ahorro de Energía, Madrid: Ministerio de Industria, Comercio y Turismo.

Jiménez M.A. (2008). Integración de los procesos agronómicos e hidráulicos del riego a presión en un entorno SIG para la gestión eficiente de Comunidades de Regantes. Grupo REDHISP, Instituto de Ingeniería del Agua y Medio Ambiente, Valencia: Univesitat Politècnica de València.

WWAP (2014). The United Nations World Water Development Report 2014: Water and Energy. United Nations World Water Assesment Programme, Paris: UNESCO. 